

DOI: http://doi.org/10.48195/jie2021-067

\title{
PROMOÇÃO DA FUNCIONALIDADE DO IDOSO INSTITUCIONALIZADO NO MEIO VIRTUAL DURANTE A PANDEMIA DA COVID-191
}

\author{
Stéphani Sortica Fantinel ${ }^{2}$; Carolina Araujo Londero³; Munah Najeh Saleh Ahmad \\ Maruf $^{4}$;Nathália Bordin Mendes ${ }^{5}$; Naiana Oliveira dos Santos ${ }^{6}$; Maria Helena \\ Gehlen ${ }^{7}$.
}

\section{RESUMO}

O envelhecimento caracteriza-se como sendo um processo natural, universal e irreversível, devido ao aumento da expectativa de vida e diminuição da natalidade. Objetiva-se descrever a manutenção e promoção da funcionalidade do idoso institucionalizado durante a pandemia COVID-19 ao se utilizar o meio virtual. Trata-se de um relato de experiência, construído ao longo do segundo semestre de 2020, durante as adequações das ações extensionistas da disciplina de Atenção Integral à Saúde do Idoso da Universidade Franciscana. Sendo realizadas videochamadas, aniversário virtual e cards, em uma instituição de longa permanência de idosos, no interior do Rio Grande do Sul. A vivência possibilitou o protagonismo empreendedor, virtualmente, durante a pandemia ao reduzir o sentimento de solidão e quadros depressivos, para isso, tenciona-se a permanência do afeto, vínculo e amorosidade, já que o estímulo às pessoas idosas, em suas atividades de vida diária, se relaciona com esperança, conforto e autoestima na manutenção da funcionalidade.

Palavras-chave: Envelhecimento; Infecções por Coronavirus ; Promoção da Saúde.

\begin{abstract}
Aging is characterized as a natural, universal, and irreversible process due to the increase in life expectancy and decrease in birth rate. The objective is to describe the maintenance and promotion of the functionality of the institutionalized elderly during the COVID-19 pandemic by using the virtual environment. This is an experience report, built during the second semester of 2020, during the adjustments of the extension actions of the discipline of Comprehensive Care of Elderly Health at the Franciscan University. We carried out video calls, virtual birthday and cards, in a long-stay institution for the elderly, in the countryside of Rio Grande do Sul. The experience made possible the entrepreneurial protagonism, virtually, during the pandemic to reduce the feeling of loneliness and depressive conditions, for this, it is intended the permanence of affection, bonding and lovingness, since the stimulus to the elderly, in their activities of daily living, relates to hope, comfort and self-esteem in maintaining functionality.
\end{abstract}

Key Words: Aging; Coronavirus Infections; Health Promotion. 


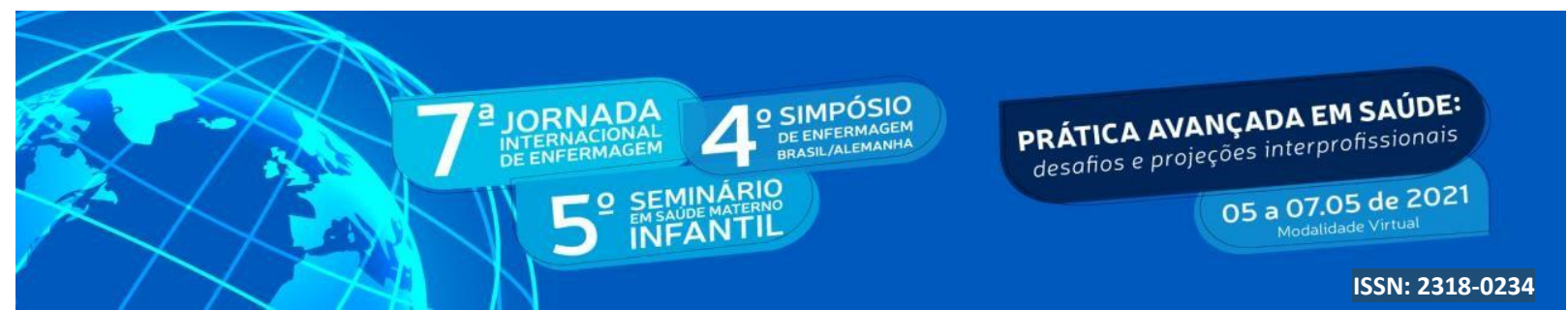

${ }^{1}$ Relato de experiência. Promoção Da Funcionalidade Do Idoso Institucionalizado No Meio Virtual Durante A Pandemia Da Covid-19.

2 Discente de Enfermagem da Universidade Franciscana. Email: stephanifantinel@gmail.com. 3Discente de Enfermagem da Universidade Franciscana Email: carolina.alondero@gmail.com. ${ }^{4}$ Discente de Enfermagem da Universidade Franciscana Email: munahsaleh1999@ gmail.com.

${ }^{5}$ Discente de Enfermagem da Universidade Federal de Santa Maria. Email: nathaliabordinm@ gmail.com.

${ }^{6}$ Docente. Orientadora. Dr ${ }^{\mathrm{a}}$ em Enfermagem pelo Programa de Pós-Graduação em Enfermagem da

Universidade Federal do Rio Grande do Sul. Email: naiana.santos@ufn.edu.b.

${ }^{7}$ Docente. Orientadora. Dr ${ }^{\mathrm{a}}$ em Gerontologia Biomédica pela Escola de Medicina da Pontifícia Universidade Católica do Rio Grande do Sul, docente da Universidade Franciscana. Email: gehlenmh@gmail.com.

\section{INTRODUÇÃO}

O crescente e acelerado processo do envelhecimento, principalmente quando abordado a população brasileira, sendo notável através dos índices do Instituto Brasileiro de Geografia e Estatística que estimam que haja em média 13,4\% de pessoas com sessenta anos ou mais (IBGE, 2018). Nesse sentido, é necessário reconhecer as mudanças fisiológicas significativas que aparecem conforme o indivíduo envelhece.

$\mathrm{O}$ envelhecimento humano caracteriza-se por um processo natural, universal e irreversível, nesse sentido, o aumento da expectativa de vida no Brasil relaciona-se a um fenômeno mundial. Sendo assim, a nível biológico, o envelhecimento é associado ao acúmulo de uma grande variedade de efeitos moleculares e celulares, com o decorrer dos anos, ocasiona uma perda gradual e implica em alterações nas reservas fisiológicas do indivíduo, assim como, a uma elevação do risco de contrair diversas patologias, bem como, um declínio geral na capacidade intrínseca do indivíduo (OMS, 2015 e GEHLENPEREIRA, 2018).

Nesse sentido, a pessoa idosa ao envelhecer em uma instituição de longa permanência de idosos -ILPI-necessita estimular e manter a sua funcionalidade durante o contexto epidêmico, pois apresenta estreita associação com o desenvolvimento da fragilidade. A fragilidade configura um estado de saúde acometido pela baixa tolerância 


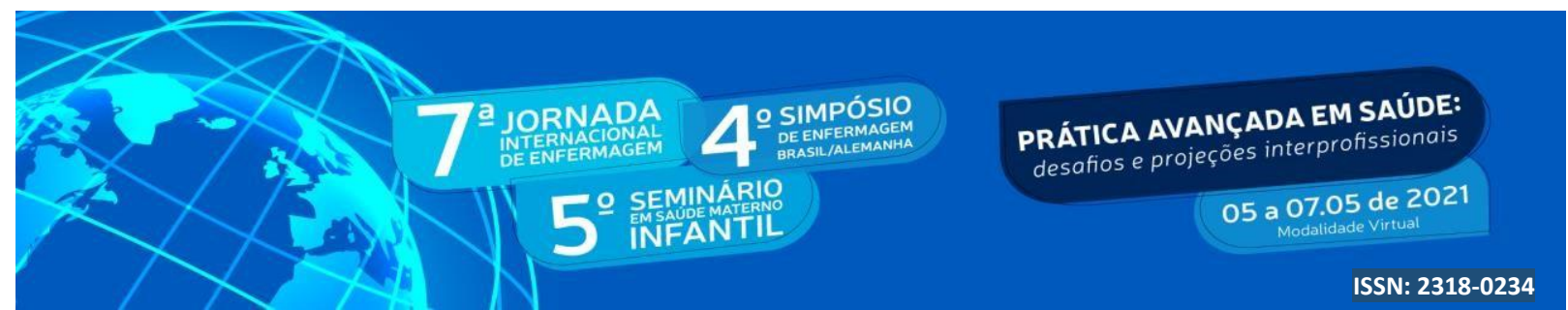

aos estressores físicos e psicológicos, o que acarreta no aumento do número de acidentes, como quedas, o declínio da capacidade funcional, a dependência nas atividades básicas de vida diária, e em cinco anos, leva-se a um prognóstico de morte antecipada (LENARDT et al., 2016).

O contexto epidêmico originado pelo agente infeccioso, coronavírus, originou-se de uma família de vírus responsáveis por causar síndromes respiratórias que podem ser leves a moderadas, no entanto tornou-se notório o aumento de casos confirmados com problemas respiratórios graves sendo as pessoas idosas consideradas população vulnerável ou grupo de risco à infecção pela COVID-19, e sobretudo, idosos que residem em ILPI (BARBOSA, et al, 2020).

De tal modo, o meio virtual utilizado na promoção da saúde contribui para a manutenção e promoção da funcionalidade da pessoa idosa institucionalizada, pois idosos vulneráveis residente em ILPI, necessita de atenção especializada em preservação ao seu bem-estar e vitalidade.

\section{OBJETIVO}

Descrever a manutenção e promoção da funcionalidade da pessoa idosa institucionalizada durante a pandemia ao se utilizar o meio virtual.

\section{METODOLOGIA}

O presente artigo trata-se de um relato de experiência construído ao longo do segundo semestre de 2020, entre o período de julho a dezembro para a disciplina de Atenção Integral à Saúde do Idoso, do urso de Graduação em Enfermagem da Universidade Franciscana, juntamente com o projeto extensionista "Educação Interprofissional: a promoção do bem-estar e da funcionalidade em idosos”. Sendo que, as ações ocorriam em uma Instituição de Longa Permanência de Idosos localizada em um município central do interior do estado do Rio Grande do Sul. 


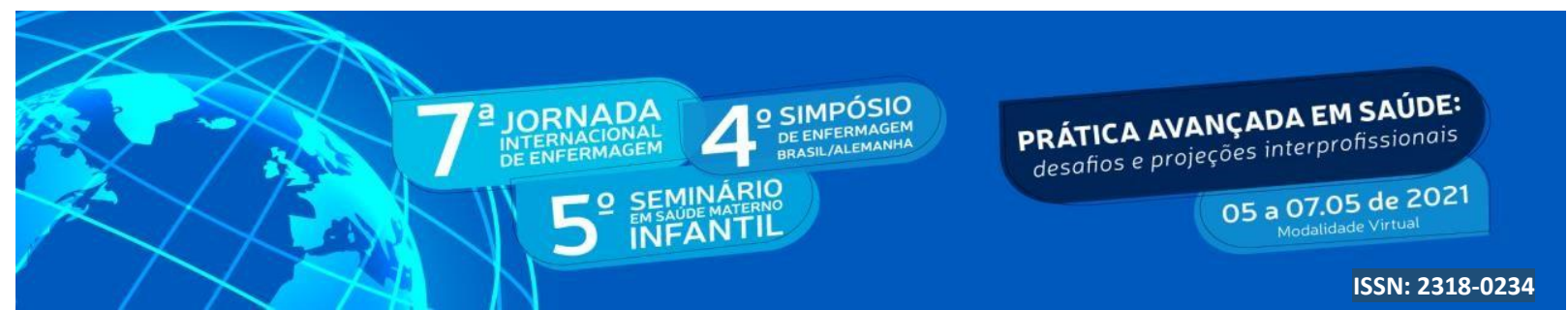

Para tanto, a base de dados utilizada foi a Biblioteca Virtual em Saúde e consultas aos bancos de dados Scientific Electronic Library Online e Literatura Latino-Americana e do Caribe em Ciências da Saúde.

As ações extensionistas outrora presenciais foram readequadas para a modalidade virtual por meio de vídeo chamadas, cards, arrecadações de donativos de modo virtual, aniversários virtuais, mensagens e cartas virtuais, além da criação de uma conta em uma rede social, cujo intuito é informatizar e levar até a população as atividades exercidas frente a pandemia do COVID -19, além de promover maior alcance para arrecadação de alimentos e itens básicos de higiene, para as idosas residentes na ILPI.

\section{RESULTADOS E DISCUSSÃO}

Com a vulnerabilidade da pessoa idosa aumentada no contexto pandêmico, nos mais variados aspectos da vida, o meio virtual para efetivas as ações extensionistas colaborou de forma a garantir conforto e estimular a funcionalidade com melhoria do processo de envelhecimento e no envelhecer na ILPI.

Nesse âmbito salienta-se que, além das mudanças fisiológicas que ocorrem em decorrência do avanço da idade, existem também síndromes que podem atingir o idoso e para além disso, as síndromes geriátricas, ou seja, fatores multidimensionais que influenciam nos aspectos sociais, familiares, clínicos e funcionais da pessoa idosa (GEHLEN-PEREIRA,2018).

As videochamadas, realizadas as assistidas, efetivaram a promoção e manutenção da funcionalidade no contexto pandêmico, por meio do vínculo e estímulo às atividades de vida diária. Neste contexto, observa-se que a pessoa idosa institucionalizada começa a ter outro cotidiano principalmente em tempos de pandemia pela covid 19. Além disso, percebe-se através da vivência o distanciamento e o isolamento social, além das rotinas dispostas pelos horários para medicações, banho, lazer, alimentação o que direcionaram novas as abordagens do atendimento geriátrico e gerontológico na ILPI, na promoção 


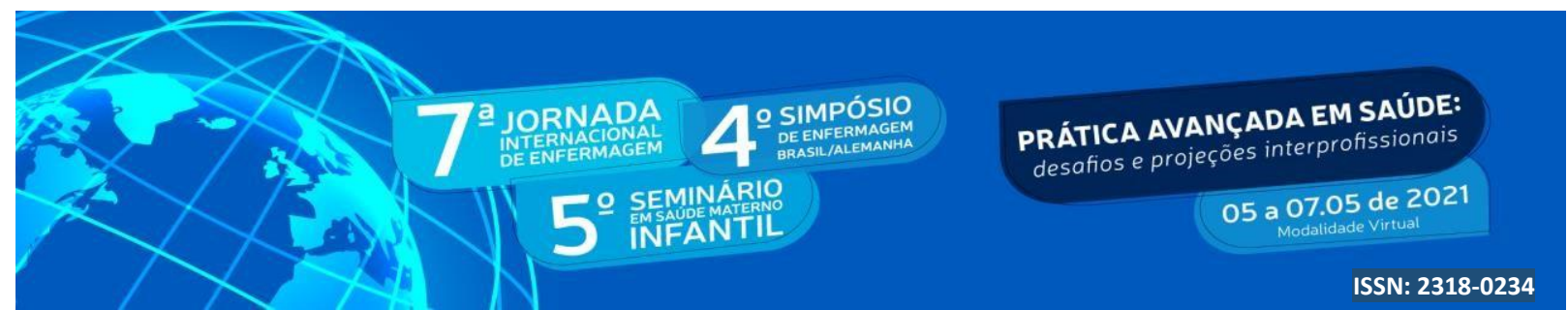

da saúde e bem-estar com funcionalidade no contexto de vida cotidiana e pandêmica em razão ao coronavírus.

$\mathrm{Na}$ transição da ação extensionista presencial para a virtual, foi realizado uma anamnese e assim, sendo possível conhecer os principais aspectos que careciam de diligência. Para tanto, através do contato com profissionais da saúde da ILPI, alicerçouse as necessidades. E, com um processo de reinvenção marcado por um período repleto de incertezas, consolidou-se o vínculo com as idosas de uma Instituição de Longa Permanência de Idosos por meios virtuais de comunicação (VELHO-HERÉDIA, 2020).

Desse modo, integrado o principal meio de comunicação - vídeos chamadas-, destarte criado uma rede social com informações e orientações sobre o idoso e o cuidado com o mesmo, além de envio de cards, aniversário virtual e donativos, com o foco em preservar a autonomia do sujeito, bem estar e funcionalidade, principalmente com estímulo cognitivo. Outrossim, durante atividades virtuais descritas, obtiveram-se um resultado positivo no que tange o mantimento do laço, além da preservação da funcionalidade das pessoas idosas institucionalizadas .

Embora as ILPI no Brasil mantenham-se distantes do arquétipo estabelecido, as mesmas estão em constante procura de adequação às normativas existentes. As instituições colaboram por uma parte significativa, sendo sua função precordial acolher pessoas idosas, que por incontáveis vezes encontram-se desamparadas pelas suas famílias ou por vontade própria do indivíduo de habitar em uma instituição, bem como, outros motivos que levam eles a serem incluídos no grupo de idosos institucionalizados (GATO, et al, 2019).

O foco ao realizar essa comunicação virtual está voltado para a preservação da vitalidade e funcionalidade, como cerne nas atividades de vida diária-AVD e nas atividades instrumentais de vida diária de AIVD. A Organização Mundial da Saúde define 65 anos de idade, o marco da maximização da prevalência de problemas específicos que englobam o envelhecimento, como por exemplo a diminuição da 


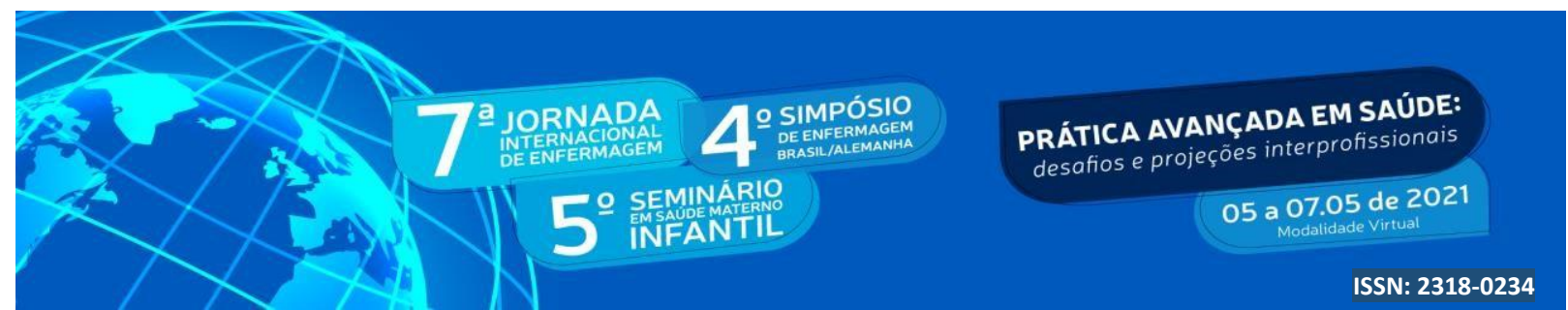

acuidade visual e auditiva, incontinências, incidências de quedas, demência, depressão e isolamento social (OMS, 2015).

Além disto, por compreender o conjunto de fatores que englobam os idosos da ILPI, tais como, diminuição da autoestima, solidão, isolamento social, perda da identidade e liberdade, dellirium, demência e depressão. A comunicação estabelecida com as idosas possibilitou uma elevação da autoestima, estímulo da funcionalidade e desenvolvimento de atividades diárias sem ou com mínimo auxílio GEHLENPEREIRA,2018).

Outrossim, contribui para a redução do sentimento de solidão e quadros depressivos, para isso, tenciona-se a permanência do afeto, elo e amorosidade, fez-se possível o manejo adequado para cada idosa, com base na humanização com base na Política Nacional da Humanização - PNH, através de um cuidado singular (BRASIL, 2013).

Ao compreender a PNH e o PTS, ambos estimulam a comunicação, humanização e a autonomia do sujeito, para tanto para realizar o PTS, faz-se necessário considerar a PNH que compreende a respeito do acolhimento, com escuta qualificada e comunicação terapêutica, do assim como, a ambiência, corresponsabilidade e autonomia, entre outros (BRASIL, 2013). Dessa maneira, o PTS é um conjunto de ideias terapêuticas, propostas ao indivíduo e/ou a família, que resulta da discussão coletiva e discussão em equipe interdisciplinar (BRASIL, 2013 e TRAD-ROCHA, 2011).

Nesse contexto, a vivência possibilita compreender a senescência, bem como, a senilidade decorrente do processo desgastante em consequência da pandemia. Ademais, pela carência de afeto, distanciamento dos entes queridos e isolamento social, desse modo, a comunicação terapêutica on-line com as institucionalizadas. Tendo em vista, as melhorias obtidas através do contato, com a preservação de um idoso robusto com riscos diminuídos de fragilidade, preservação da funcionalidade, estímulo à autonomia e sendo possível promover e educar em saúde de modo eficaz. 


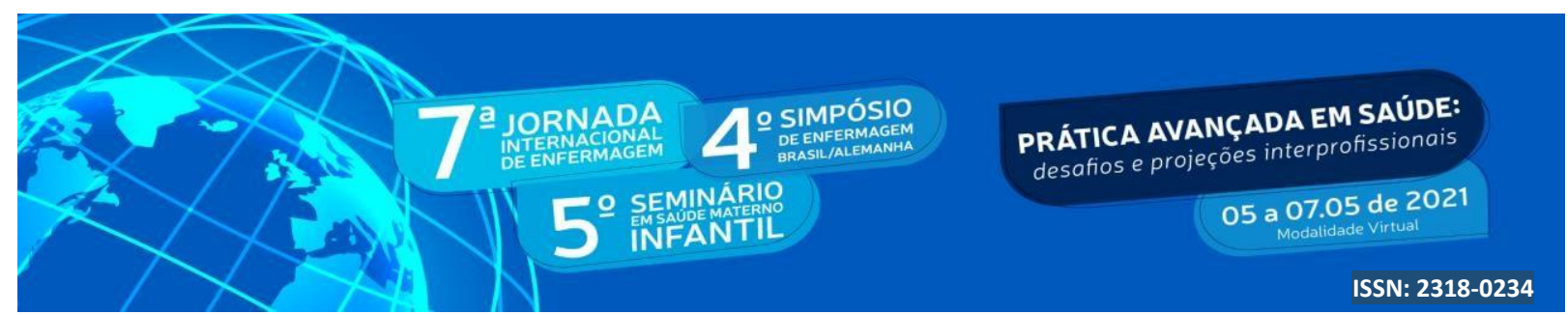

\section{CONCLUSÃO}

À vista isto, a descrição da manutenção e promoção da funcionalidade da pessoa idosa institucionalizada durante a pandemia ao se utilizar o meio virtual mostrou que é possível estabelecer vínculo, instigar, estimular a preservação do exercício das atividades diárias humana básicas e instrumentais fundamentais para o idoso. Outrossim, manter e promover a funcionalidade do idoso institucionalizado.

\section{REFERÊNCIAS}

BARBOSA, Andreia Clerice et al. Atenção ao idoso frente à pandemia por Covid-19. Revista de Saúde Pública do Paraná, v. 3, n. Supl., 2020

BRASIL. Ministério da Saúde. Política Nacional da Humanização. Brasília, 2013.

GATO, Jussara Marília et al. Saúde mental e qualidade de vida de pessoas idosas. Avances en Enfermería, v. 36, n. 3, p. 302-310, 2018.

GEHLEN, Maria Helena; PEREIRA, Adriana Dall'Asta. Enfermagem em Gerontologia. $1^{\mathrm{a} e d}$. Porto Alegre, RS: Moria,2018.190p.

INSTITUTO BRASILEIRO DE GEOGRAFIA E ESTATÍSTICA. Projeção da população do Brasil e das Unidades da Federação-Pirâmide etária 2010-2060. Brasília, 2018.

LENARDT, Maria Helena et al. Fragilidade e qualidade de vida de idosos usuários da atenção básica de saúde. Revista Brasileira de Enfermagem, v. 69, n. 3, p. 478-483, 2016.

ORGANIZAÇÃO MUNDIAL DA SAÚDE. Relatório mundial de envelhecimento e saúde. Estados Unidos, v. 30, p. 12, 2015.

TRAD, Leny Alves Bomfim; ROCHA, Ana Angélica Ribeiro de Meneses. Condições e processo de trabalho no cotidiano do Programa Saúde da Família: coerência com princípios da humanização em saúde. Ciência \& Saúde Coletiva, Rio de Janeiro, v.16, n.3, p. 1969-1980, 2011.

VELHO, FÁBIO DANIEL; HERÉDIA, VANIA BM. O Idoso em Quarentena e o Impacto da Tecnologia em sua Vida. Rosa dos Ventos, v. 12, n. 3, p. 1-14, 2020. 